Gynäkologe 2010 • 43:111-112

DOI 10.1007/s00129-009-2492-x

Online publiziert: 27. Januar 2010

๑) Springer-Verlag 2010

K. Diedrich ${ }^{1} \cdot$ K. Friese $^{2} \cdot$ M. Thill ${ }^{1} \cdot$ W. Jonat ${ }^{3}$

${ }^{1}$ Klinik für Frauenheilkunde und Geburtshilfe, Universitätsklinikum Schleswig-Holstein, Campus Lübeck

${ }^{2}$ Ludwig-Maximilians-Universität München, Standorte Innenstadt, Großhadern, München

${ }^{3}$ Klinik für Gynäkologie und Geburtshilfe, Universitätsklinikum Schleswig-Holstein, Campus Kiel

\title{
Entwicklungen in der endoskopischen Gynäkologie
}

zur Therapie der tubaren Sterilität. Durch die Etablierung der assistierten Reproduktion (ART) ist die Expertise in der operativen Tubenrekonstruktion rückläufig. Daher fokussiert dieser Beitrag neben der ART auch auf die verschiedenen operativen Möglichkeiten. Bis heute gibt es bedauerlicherweise keine prospektiv randomisierte Studie, die beide Methoden miteinander verglichen hat. mend komplexere operative griffe. Um einen Ausbildungsstandard in der gynäkologischen Endoskopie zu sichern, ist das Ausbildungskonzept der Arbeitsgemeinschaft Gynäkologische Endoskopie e.V. (AGE) der Deutschen Gesellschaft für Gynäkologie und Geburtshilfe e.V. (DGGG) in Deutschland mittlerweile fest implementiert. Durch die rasante Geschwindigkeit der medizintechnischen Entwicklung steht dem Operateur mittlerweile ein Instrumentarium zur Verfügung, mit dem auch neue, weniger invasive und narbenfreie Operationstechniken möglich sind. Das vorliegende Heft befasst sich eingehend und sehr ausführlich mit ausgesuchten Themen der gynäkologischen Endoskopie und stellt sowohl die Standards als auch die aktuellen Entwicklungen dar.

Zunächst wird das kontrovers diskutierte Thema der Adhäsionsprophylaxe referiert. Dabei werden die zum Einsatz kommenden verschiedenen Substanzen und deren Sinn und Nutzen diskutiert. Der daran anschließende Beitrag beschreibt die verschiedenen Methoden

\section{(7) Heute sind auch onkologisch radikale Operationen sicher laparoskopisch möglich}

Bei einigen Operationen, wie der Hysterektomie, haben die endoskopischen Techniken das Operieren am offenen Bauch definitiv in den Hintergrund gedrängt. In diesem Heft wird speziell auf die Methode der laparoskopischen suprazervikalen Hysterektomie (LASH) eingegangen, Vorund Nachteile dieser Methode werden vor dem Hintergrund der aktuellen Datenlage dargestellt.

Der darauf folgende Beitrag arbeitet die Vorteile des laparoskopischen Staging beim Zervixkarzinom heraus und erläutert praxisorientiert und detailliert die operativen Schritte der laparoskopisch radikalen Hysterektomie. In der OnlinePublikation findet der Leser dazu verschiedene Videosequenzen, die das operative Vorgehen noch einmal plastisch kommentieren. Durch die Weiterentwicklung der endoskopischen Operationstechniken ist es mittlerweile möglich, nicht nur onkologisch radikale Operationen sicher durchzuführen, sondern, bedingt durch die Möglichkeit der optischen Vergrößerung, auch sehr filigrane Strukturen zu erkennen. Der Beitrag zur nervenschonenden laparoskopischen radikalen Hysterektomie erklärt anschaulich, wie die Identifikation der autonomen Nervenversorgung von Blase und Rektum zu gewährleisten ist und wie es auf diese Weise gelingt, die operationsbedingte Morbidität deutlich zu reduzieren. Da die radikale Operation des Zervix- oder Korpuskarzinoms in der Regel nicht ohne eine pelvine und/oder paraaortale Lymphonodektomie durchgeführt werden sollte, ist diese auch beim laparoskopischen Vorgehen zu praktizieren. Daher ist dieser Operation ein eigener Beitrag gewidmet, in dem die aktuelle Studienlage diskutiert und ausführlich bewertet wird.

\section{(? Die Akzeptanz endoskopischer Operationen führt zur Entwicklung noch „,weniger invasiver" Methoden}

Die hohe Patientinnenakzeptanz endoskopischer Operationen führt zur Entwicklung neuer, „weniger invasiver“ Techniken, von denen in zwei Arbeiten je eine beschrieben wird. Die erste dieser Arbeiten beschäftigt sich mit der LESS (LaparoEndoscopic Single-Site)-Technik, einer Technik, die einen einzigen Trokar für 
Kamera und Instrumente verwendet. Die zweite Arbeit erklärt und erläutert eine weitere vielversprechende Entwicklung der Endoskopie, die NOTES (Natural Orifice Transluminal Endoscopic Surgery)Technik, die über einen transgastrischen oder transvaginalen Zugang operative Eingriffe am inneren Genitale ermöglicht. Ob sich eine - und welche - oder beide dieser neuen Techniken durchsetzen werden, ist noch unklar.

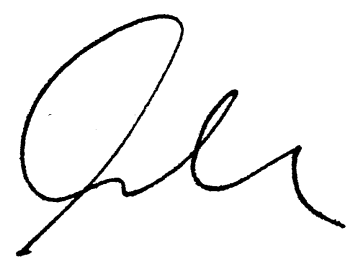

Prof. Dr. K. Diedrich

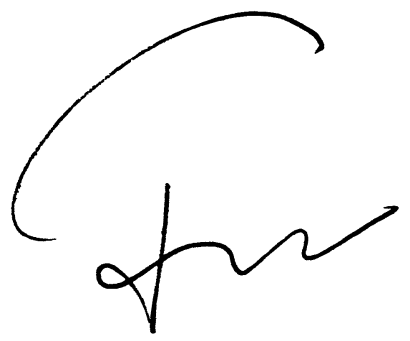

Prof. Dr. K. Friese

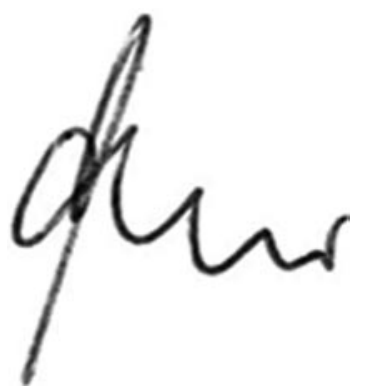

Dr. M. Thill

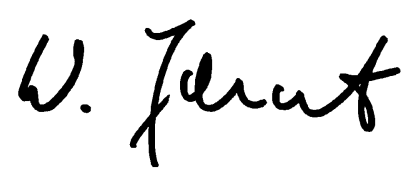

Prof. Dr. W. Jonat

\section{Korrespondenzadresse}

Prof. Dr. K. Diedrich

Klinik für Frauenheilkunde und Geburtshilfe, Universitätsklinikum Schleswig-Holstein, Campus Lübeck Ratzeburger Allee 160, 23562 Lübeck klaus.diedrich@uk-sh.de
Der Gynäkologe bietet jeden Monat umfassende und aktuelle Beiträge zu interessanten Themenschwerpunkten aus allen Bereichen der Gynäkologie und Geburtshilfe.

Möchten Sie ein bereits erschienenes Heft nachbestellen? Die folgenden Ausgaben aus 2009 und bereits erschiene Ausgaben aus 2010 können Sie direkt bei unserem Kundenservice zum Preis von EUR 33,- beziehen:
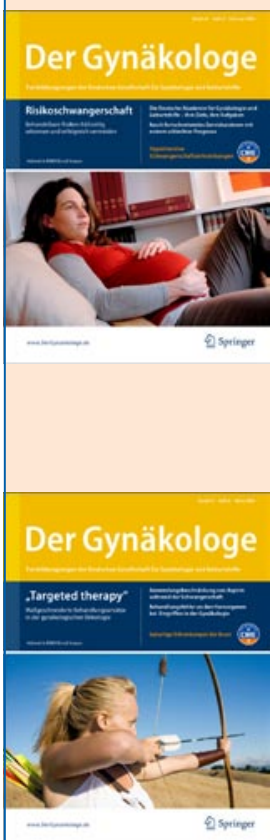

Leitthemen in Der Gynäkologe 2009

- 01/09 Habituelle Aborte

- 02/09 Risikoschwangerschaft

- 03/09 Targeted Therapy

- 04/09 Erkrankungen von Vulva und Vagina

- 05/09 40 Jahre Cardiotokographie

- 06/09 Neue Aspekte der Strahlentherapie in der Gynäkologie

- 07/09 Reproduktionsmedizin und Embryonenschutz

- 08/09 Verhalten in kritischen Rechtssituationen

- 09/09 Mammakarzinom in besonderen Situationen

- 10/09 Infektionserkrankung in Gynäkologie und Geburtshilfe

- 11/09 Prädiktive Diagnostik in der Frauenheilkunde

- 12/09 Zervixkarzinom

\section{Leitthemen in Der Gynäkologe 2010}

- 01/10 Verhalten in kritischen Rechtssituationen, Teil II

- 02/10 Innovationen in der minimal-invasiven Chirurgie der Gynäkologie

- 03/10 Zentrumsbildung in der Gynäkologie und Geburtshilfe

- 04/10 Dokumentationssysteme in der Frauenheilkunde

- 05/10 HRT und neue Leitlinie

- 06/10 Medizin am Rand der Lebensfähigkeit, zwischen Schicksal und Groningen-Protokoll

- 07/10 Molekularbiologische Mechanismen für den Kliniker

- 08/10 Grenzsituationen in der Urogynäkologie

- 09/10 Frauenärztin/Frauenarzt der Zukunft

- 10/10 Psychosoziale Aspekte der Gynäkologie

- 11/10 Endometriose

- 12/10 Mammakarzinom - Neue Aspekte aus Praxis und Politik

(Änderungen vorbehalten)

So erreichen Sie unseren Kundenservice:

Springer Medizin Verlag GmbH

Kundenservice Zeitschriften

Haberstraße 7

69126 Heidelberg

Tel. +496221 345-4303

Fax: +496221 345-4229

E-Mail: subscriptions@springer.com

www.DerGynaekologe.de 